\title{
LOS INTÉRPRETES GENERALES DE YUCATÁN: HOMBRES ENTRE DOS MUNDOS
}

\author{
MARK LENTZ
}

\begin{abstract}
Resumen: Este artículo se centra en un tipo importante de intermediarios en el Yucatán colonial, los intérpretes generales del Juzgado Privado de Indios de Yucatán. Desde la época de Gaspar Antonio Chi en 1580 hasta la desaparición de esta corte en la década de 1820 , estos hombres mediaron directamente en disputas de propiedades, quejas contra las autoridades, y tradujeron decretos inicialmente publicados en español para la mayoría maya hablante de la provincia. Sus actividades demuestran que Yucatán poseyó un alto número de individuos que dominaron tanto el maya como el español hasta finales del periodo colonial. Además, la cantidad de individuos calificados sirviendo extraoficialmente como intérpretes durante finales del siglo dieciocho, y el sorprendente número de nomayas que necesitaron sus servicios, muestran también que el periodo colonial fue una época en la que el maya se extendió como lenguaje predominante en Yucatán, en lugar de una época de declive para ese idioma.
\end{abstract}

AвSTRACT: This paper focuses one type of important intermediaries of colonial Yucatan, the general interpreters of Yucatan's Juzgado Privado de Indios. From the time of Gaspar Antonio Chi in 1580 until the demise of this court in the 1820 s, these men mediated directly in land disputes, complaints against priests and other officials, and translated decrees initially published in Spanish for the province's Maya-speaking majority. The activities of the interpreters demonstrate that Yucatan had a high number of individuals literate and fluent in both Maya and Spanish until the close of the colonial period. Furthermore, the quantity of qualified individuals serving unofficially as interpreters during the late eighteenth century and the surprising number of non-Mayas in need of their services also shows that the colonial period was a time in which Maya spread as the predominant language of Yucatan rather than a time of decline for the region's native language.

Palabras Clave: intérpretes, intermediarios, Chikindzonot, bilingiies, castas.

KEY WORDS: interpreters, intermediaries, Chikindzonot, bilingual groups, castes.

RECEPción: 25 de enero de 2008.

ACEPTACIÓN: 19 de febrero de 2008. 



\title{
LOS INTÉRPRETES GENERALES DE YUCATÁN: HOMBRES ENTRE DOS MUNDOS*
}

\author{
MaRK LenTZ \\ Tulane University
}

Un concepto dominante en la historiografía del Yucatán colonial, hasta hace unos diez años, más o menos, ha sido la idea de la existencia de una segregación rígida entre la mayoría indígena y la minoría española. Sin embargo, dicho concepto ignora la profunda influencia linguiística que se ha dado entre los idiomas español y maya, y a la vez no reconoce la importancia de las numerosas personas que no habrían podido ser incluidas con facilidad en ninguna de las dos repúblicas propuestas, ni en la de los españoles, ni en la de los indios. ${ }^{1}$ Nancy M. Farriss, por ejemplo, quien propuso la existencia de un "Muro de Berlín" entre estos dos sectores de la población, supone que entre ellos prácticamente no se producía ningún contacto, aunque en la realidad dicha separación no existía. ${ }^{2}$

La presencia e importancia de otros grupos ha sido bien investigada en libros escritos por Francisco Fernández Repetto, Genny Negroe Sierra, Melchor Campos García, Jorge Victoria Ojeda, Jorge Canto Alcocer, y Matthew Restall. ${ }^{3}$ Además, Restall y especialmente Wolfgang Gabbert han modificado la idea de que los

\footnotetext{
- Agradezco mucho a María de los Dolores de Miguel por su apoyo en la redacción de este artículo.

${ }^{1}$ Teóricamente, la sociedad de las Américas se encontraba dividida legal y geográficamente entre los indigenas y los españoles - dos repúblicas - a partir de la conquista.

${ }^{2}$ Farriss, Maya Society under Colonial Rule: The Collective Enterprise of Survival. Princeton, N. J. Princeton University Press, 1984, p. 166 (En la versión española, publicada en 1992, no apareció esta frase, pero Farriss usó la exagerada frase en su original escrito en inglés.)

${ }^{3}$ Victoria Ojeda y Canto Alcocer, San Fernando Aké: Microhistoria de una comunidad afroamericana en Yucatán. Mérida: UADY, 2006, Campos García, Castas, feligresía y ciudadanía en Yucatán: los afromestizos bajo el régimen constitucional español, 1750-1822. Mérida: CONACYT/UADY, 2005. y Restall, The Black Middle (forthcoming) son los libros más recientemente publicados sobre los yucatecos de herencia africana, una tendencia orientada por Fernández Repetto y Negroe Sierra en Una población perdida en la memoria: los negros de Yucatán. Mérida: UADY, 1995. La inclusión de este grupo no bien representado hasta recientemente muestra las limitaciones de ver Yucatán como una dicotomía. También merecen mención Antochiw y Espejo-Ponce Hunt, en Milicia de Yucatán (siglos xv y xvi) \& La unión de armas de 1712. México: Instituto Nacional de Antropología e Historia, CONACULTA, 2006. Antochiw observó la existencia e importancia de las divisiones de milicias pardas en su papel de defensa de Yucatán desde los primeros años de la época colonial. Espejo Ponce-Hunt, Colonial Yucatán: Town and Region in the Seventeenth Century. Ph.D. Diss, UCLA, 1974, incluyó los afroyucatecos y otras personas de ascendencia mezclada en el siglo xvi como una población destacada de Mérida.
} 
mayas eran un grupo unido. Gabbert ha observado que tanto a finales de la época colonial como durante los años anteriores a la Guerra de Castas, el uso del idioma maya no se correspondía exactamente con una ascendencia indígena, ya que algunos mayas hablaban español, en tanto que muchas más "castas", o grupos de personas de ascendencia mezclada, afroyucatecos, e incluso algunos españoles, hablaban mayormente maya y no castellano. ${ }^{4}$

Rugeley y Gabbert han encontrado muchos vínculos políticos y personales no conflictivos entre mayas y españoles, que invalidan una vez más la idea del "Muro de Berlín" de Farriss. Rugeley también ha destacado la importancia del clero rural, el cual constituyó otro grupo de intermediarios. Según este autor, la persistente presencia de este grupo fue base de estabilidad en el campo. ${ }^{5}$ Asimismo, Gabbert, al escribir sobre la Guerra de Castas, y advertir la amplia presencia de mayas luchando contra la rebelión, minimizó la importancia de las diferencias raciales como causa básica de la insurgencia, y al mismo tiempo señaló el gran número de mestizos, e incluso españoles, que lucharon del lado de los insurgentes encabezados por los batabes del este, como otro ejemplo de las limitaciones de la explicación racial como raíz del conflicto. ${ }^{6}$ En otro libro próximo a publicar, Restall ha explorado el papel de los yucatecos negros, los cuales también pasaron sus vidas en contacto con ambos grupos, españoles y mayas. ${ }^{7}$ Siguiendo con este tema, el presente artículo tratará acerca de otro grupo de españoles que estuvo frecuentemente en contacto con los indígenas, y además poseyó un buen conocimiento del idioma indígena escrito y hablado: los intérpretes.

Fariss a su vez ha escrito que no existe un buen método para medir la influencia cultural recíproca entre dos grupos étnicos; sin embargo, estudios filológicos y linguíísticos revelan gran cantidad de palabras asimiladas en los dos idiomas de los que hablamos, lo que muestra un ejemplo claro de interacción cultural. Entre estos estudios se encuentran los trabajos de Alfredo Barrera Vásquez, que han desvelado numerosas palabras mayas y pronunciaciones afectadas

\footnotetext{
"Restall, en "Maya Ethnogenesis," The Journal of Latin American Anthropology 9, núm. 1 (Spring 2004): 64-89, explora la falta de una identidad "maya" que uniera a la población indígena de Yucatán durante la época colonial. Gabbert, en Becoming Maya: Ethnicity and Social Inequality in Yucatán since 1500. Tucson: University of Arizona, 2004 y también en su artículo "Of Friends and Foes: The Caste War and Ethnicity in Yucatan" The Journal of Latin American Anthropology 9, núm. 1 (Spring 2004): 90-118 empieza con el mismo punto de partida, pero lo adelanta en términos de distinguir entre el idioma hablado y etnicidad, y niega la creación de una identidad maya como resultado de la mencionada guerra, y la presencia de muchos rebeldes no mayas en la misma.

${ }^{5}$ Rugeley, Yucatán's Maya and the Origins of the Caste War. Austin: University of Texas Press, 1996, p. 32. También apuntó la posibilidad de convergencia entre el clero y la elite maya como conservadores contra ideas liberales por ser beneficiarios de obvenciones y privilegios tradicionales.

${ }^{6}$ Gabbert, "Of Friends and Foes", p. 99. Dumond también ha observado la presencia de "vecinos" entre las filas insurgentes, subrayando el aspecto de inquietud rural más que la unidad étnica de los mayas. Dumond, The Cross and the Machete: Campesino Rebellion in Yucatan. Lincoln: University of Nebraska Press, 1997.

7 Restall, The Black Middle (en prensa).
} 
por el maya, en el castellano hablado actualmente en Yucatán. Barrera Vásquez ha descrito esta zona como "un medio bilingüe desde hace cuatrocientos años", lo que ha dado como resultado un "maya llamado 'amestizado"', y el "español yucateco". El reconocido lingüista de Yucatán describió cómo el idioma maya ha afectado profundamente al español vulgar en el léxico, la sintaxis, y aún en la fonética, hasta el punto de que los fonemas se asemejan mucho en los dos idiomas, existiendo incluso glotalizadas en el español yucateco, igual que en el maya. ${ }^{8}$ Restall también ha descrito minuciosamente la adopción gradual de términos españoles por el idioma maya que se produjo durante toda la Colonia, y demostró que estas dos culturas no se encontraban aisladas una de otra. En contraposición a lo escrito por Farriss, Restall ha explicado en qué medida el estudio de un idioma puede utilizarse como un "barómetro de aculturación", método usado con éxito por James Lockhart y Frances Karttunen con la lengua náhuatl, y que él mismo utilizó para entender la influencia europea en la vida cotidiana de los mayas. ${ }^{9}$ Aunque no del todo exacta, la filología se presenta como vía más o menos fiable para la medición de interacciones culturales. De este modo, algunos estudios de este tipo para el caso que nos interesa de los mayas y españoles, confirman la existencia de estas interacciones entre ambos grupos. ${ }^{10}$

Los intérpretes, que eran capaces de leer y escribir con fluidez ambos idiomas de la península, también nos sirven para mostrar la dispersión del idioma maya fuera de sus hablantes originales, puesto que posiblemente actuaron como transmisores de la influencia mutua entre estos idiomas. Debido a la abundancia de documentos que todavía se pueden encontrar en varios archivos, y que revelan el papel esencial de los intérpretes como intermediarios, hoy vamos a centrarnos en estos individuos, los cuales tuvieron un papel imprescindible durante la época colonial, en las interacciones entre la población, en su mayoría maya hablante, y los administradores de alto nivel de México y España. ${ }^{11}$

\footnotetext{
${ }^{8}$ Barrera Vásquez, Estudios lingüisticos. Mérida: Fondo Editorial de Yucatán, 1980-1981, y La lengua maya y su influencia en el español de Yucatán (manuscrito inédito, Tulane University Latin American Library Rare Books [1948].) tratan de esta influencia. Una ponencia presentada en el VII Congreso de Mayistas, julio 10, 2007, Mérida, Yucatán por Colazo Simón, "Los fenómenos glotales en el español hablado en Yucatán", trata más específicamente de las glotalizadas.

${ }^{9}$ Restall, The Maya World, pp. 293-295.

${ }^{10}$ En un estudio sobre la influencia linguística de otro grupo de América al español del Nuevo Mundo, Lipski citó dos factores en la presencia de palabras y formas de hablar transferidas desde una clase subyugada a la sociedad dominante, el peso demográfico y la frecuencia de contacto social. El alto porcentaje de mayas en Yucatán tanto como el contacto frecuente entre mayas y españoles en algunas situaciones afectaron profundamente el español yucateco. Lipski, A History of Afro-Hispanic Language: Five Centuries, Five Continents. Cambridge: Cambridge University Press, 2005, p. 8.

11 También es obvio que la necesidad de intérpretes no desapareció con la llegada de independencia de España.
} 


\section{Los primeros intérpretes ${ }^{12}$}

Desde que el puesto de intérprete general de Yucatán fuera ocupado por primera vez por Gaspar Antonio Chi, hasta finales de la época colonial, esta posición fue de la mayor importancia en las relaciones entre los gobernantes, de herencia europea y la mayoría de la población, de herencia maya. Brevemente repasaremos las raíces de la posición de intérprete general, las cuales podemos encontrarlas en los intermediarios no oficiales que sirvieron durante los primeros años de la conquista militar y espiritual. En seguida terminaremos con un estudio específico centrado en los fines del siglo xvin, una fase en que la documentación disponible nos da una buena oportunidad para observar a la sociedad con gran detalle, un cuarto de milenio después de dicha conquista de Yucatán.

Esta región fue una de las primeras tierras mesoamericanas con las que se encontraron los españoles, y una de las últimas en ser conquistada y bien explorada. La sujeción espiritual que pretendían fue una de las más rigurosamente promulgadas por los frailes franciscanos, pero también aquello a lo que con mayor determinación se resistió la población indígena. Durante la larga conquista de Yucatán, una variedad de individuos llevó a cabo de manera informal el trabajo de intérprete - a veces forzados - por lo que muchos de ellos aparecen en las memorias escritas por la mano del conquistador Bernal Díaz del Castillo. ${ }^{13}$ Este cronista describió la captura de los primeros intérpretes, más tarde bautizados como Melchor y Julián, y también cómo estos fueron llevados a La Habana, Cuba, donde los dos aprendieron castellano para servir como intérpretes en la siguiente expedición, bajo el mando de Juan de Grijalva en $1518 .^{14}$

En un incidente ocurrido posteriormente en Champotón, quedó bien claro el potencial que poseían los intérpretes, al ser los únicos bilingüies en una situación en que la fuerza invasora no podía entender nada de lo traducido. Según Díaz del Castillo, Grijalva envió a Melchor y Julián a comunicar el perdón y su deseo de paz a cambio del cese de los ataques, después de una sangrienta batalla en la que tanto los españoles como los mayas sufrieron numerosos muertos y heridos. El cronista escribió: "creemos que los indios, Julianillo y Melchorejo no habían repetido lo que les habíamos mandado, pero al contrario, algo completamente

\footnotetext{
${ }^{12}$ Los mayas prehispánicos tampoco vivian en aislamiento. En su libro The Art of the Maya Scribe. New York: Harry N. Abrams, 1998, Coe y Kerr propusieron que la palabra chilam significa "intérprete", que representado en los glifos se refiere a traductores precolombinos, y que uno de los retos de este grupo de hombres era traducir para grupos de etnias que hablaban otros idiomas y eran tributarios de varios reinos mayas ( $\mathrm{pp} .95,98$ ). También han propuesto que en este sentido "intérprete" se refiere a traductores de los dioses (pp. 219, 223). Delvendahl responde en su tesis doctoral Las sedes del poder (2006) que chilam significó únicamente traductor de lo divino, descartando la probabilidad de que chilam significara intérprete de idiomas de los humanos (p. 132).

${ }^{13}$ Díaz del Castillo, Historia verdadera de la conquista de la Nueva España, edición crítica de José Antonio Barbón Rodríguez. México: El Colegio de México, 2005.

${ }^{14}$ Díaz del Castillo notó también que ambos eran bizcos, un factor que pudo haber contribuido a su captura.
} 
distinto", demostrando así, por primera vez en una situación yucateca, la posibilidad por parte de los intérpretes de manipular las palabras. ${ }^{15}$ Los recelos sobre la veracidad de las traducciones no desaparecerían con el transcurso del tiempo.

Cuando la incursión bajo la dirección de Cortés salió de Cuba, Julián ya había muerto, probablemente una víctima más de las enfermedades traídas de Europa por los españoles. Durante aquella entrada, Melchor y una mujer anónima de Cozumel fueron los que se encargaron de traducir en la región maya. Melchor además hubo de realizar el pesado servicio de actuar como embajador de sus captores, asegurando a los habitantes de Cozumel que los bienes robados por Pedro de Alvarado, consistentes en pavos, ropa, y oro, iban a ser devueltos y que ellos no tenían ninguna razón para temer a los españoles. ${ }^{16}$

La supervivencia de dos náufragos españoles que habían salido para Darién en 1511 sufriendo un naufragio en ruta, también ayudó mucho a la expedición de Cortés. Aguilar y Gonzalo Guerrero fueron los dos únicos supervivientes de un grupo originalmente compuesto por más de diez hombres. Aguilar, natural de Écija, España, vivió como esclavo, talando leña, cavando y buscando agua, y lógicamente recibió eufórico la oportunidad de salir de esta vida de servidumbre con la partida de Cortés. Pero Guerrero, ya un cacique de guerra entre los mayas, y padre de tres hijos, rechazó la invitación de salir con Cortés. Ambos habían asimilado mucho de la cultura maya a su propia manera: Aguilar era indistinguible de los esclavos indígenas, según la narración de Díaz del Castillo, y casi había olvidado su lengua materna, mientras que Guerrero había llegado incluso al punto de tatuarse la cara y adoptar el estilo de vida de un maya perteneciente a la elite. ${ }^{17}$

Presagiando lo que iba a pasar más tarde, la tarea de traducir entre mayas y españoles pasó de manos de los indígenas a manos españolas, ya que poco después del ingreso de Aguilar como intérprete, Melchor abandonaría la expedición. Pero los españoles no dejaron de aprovecharse del apoyo indígena completamente. Más tarde, Jerónimo de Aguilar y doña Marina se combinaron para formar un equipo de traducción, trabajando juntos durante toda la conquista de México, aunque con el tiempo doña Marina es la que ha figurado de forma más notoria como intérprete, consejera y amante de Cortés.

\section{Los franciscanos y los primeros estudios lingüísticos del maya}

Siguiendo los pasos de los conquistadores de Yucatán, llegaron a la península los franciscanos, quienes tuvieron un importante papel y, según algunos, toma-

\footnotetext{
${ }^{15}$ Díaz del Castillo, Historia verdadera, p. 27.

${ }^{16}$ Ibid., pp. 41-42.

${ }^{17}$ Id.
} 
ron el control casi completo de la península. Bajo el liderazgo de los frailes, la informalidad de las traducciones improvisadas por los intérpretes provisionales cedió el paso a los estudios y a los avances en el entendimiento dirigidos por los esfuerzos de los franciscanos. Los frailes, creyendo que los glifos de los mayas estaban afiliados con la idolatría, adaptaron el idioma maya al alfabeto romano modificado, sistema que fue utilizado en la gran mayoría de los escritos de la época colonial. Gramáticas, doctrinas y diccionarios sobre el idioma autóctono de Yucatán fueron publicados por los frailes. ${ }^{18}$ También enseñaron a neófitos mayas esta nueva forma de escritura, en combinación con los estudios religiosos, y la enseñanza de los idiomas castellano y latín.

Sin traductores oficiales, los frailes franciscanos y curas seculares ocuparon el puesto de intérpretes no oficiales durante una gran parte del siglo xvı, pero a partir de 1580 aproximadamente, los franciscanos experimentaron una gradual reducción de sus poderes. Hasta este declive, además de sus estudios del idioma maya (las gramáticas, doctrinas y diccionarios), los franciscanos también tradujeron muchos de los primeros documentos escritos en la lengua original de la península. La obra mejor conocida de este género es, sin duda, la Relación de las cosas de Yucatán, reconocida tradicionalmente como una obra realizada de manera individual por el provincial de los frailes franciscanos y más tarde obispo de esa misma provincia, fray Diego de Landa. ${ }^{19}$ Supuestamente escribió esta obra en 1566 como defensa de sus celosas y violentas actividades en Maní, donde intentó terminar para siempre con las prácticas religiosas de los mayas. Sin embargo, un estudio de Matthew Restall y John Chuchiak propone que varios autores, posiblemente Francisco Domínguez, Francisco Cervantes de Salazar y Gaspar Antonio Chi, podrían haber contribuido también a esta obra sin crédito, basándose tanto en diferencias en la letra manuscrita y el papel del original, como en discrepancias de estilo inherentes a la obra. ${ }^{20}$

A pesar de las dudas sobre su supuesto autor, este libro es una obra esencial para los estudios sobre los mayas yucatecos. En este libro, un "alfabeto" de los glifos utilizados por los mayas así como las observaciones etnográficas que contiene, proporcionan una base importante para el conocimiento acerca del Yucatán precolombino y los mayas en los primeros años después de la conquista. Aunque al mismo tiempo, la quema de numerosos libros escritos a base de glifos por los mayas y de otros artefactos de la cultura material, que el mencionado fray Diego de Landa llevó a cabo, sin duda destruyó mucho más de lo que con su libro haya podido contribuir. Por último, para mostrar un ejemplo más de estas traducciones, en su libro Historia de Yucatán, el franciscano fray Bernardo

\footnotetext{
${ }^{18}$ E.g. el "Diccionario de Motul" de los fines del siglo xv, atribuido a fray Antonio de Ciudad Real, o Arte en lengua de maya y otros escritos por Juan Coronel, del mismo tiempo, o Gabriel de San Buenaventura, Arte de la lengua maya. México, 1684.

${ }^{19}$ Restall y Chuchiak IV, en "A Reevaluation of the Authenticity of Fray Diego de Landa's Relación de las cosas de Yucatán", Ethnohistory 49, núm. 3 (Summer 2002): 651-669.

${ }^{20}$ Id.
} 
de Lizana tradujo varias profecías en las cuales — según él— se presagiaba la llegada de los españoles. ${ }^{21}$

Historiadores como John Chuchiak, Inga Clendinnen y Stella María González Cicero han documentado el ascenso y auge de los frailes franciscanos como el grupo más poderoso de Yucatán durante los primeros años después de la conquista. ${ }^{22}$ El declive del poder de los clérigos resultó de enfrentamientos con encomenderos poderosos y la elite indígena, situaciones que revelaron que los franciscanos abusaron de sus neófitos indígenas y engañaron a las autoridades sobre los informes de estas infracciones. Entre otras dudas acerca de la posición prominente de los frailes, la confiabilidad de sus pretensiones de representar a los mayas imparcialmente fue puesta en duda. La veracidad de una serie de "Cartas de los indios caciques gobernadores de los pueblos de esta gobernación de Yucatán para su Magestad, escrita en lengua", que fueron traducidas por Alonso de Arévalo, un encomendero que descendía de una familia de conquistadores, como documentos escritos voluntariamente por sus autores sin ninguna presión por parte de los frailes, es cuestionable. ${ }^{23}$ En la residencia de 1561 del doctor don Diego de Quijada sirviendo como juez, declaraciones hechas en maya fueron reproducidas palabra por palabra en su idioma original, traducidas por el padre Pedro de Valencia, un sacerdote que servía como intérprete comisionado de visita. Los religiosos seculares gozaron de mayor confianza de parte de los gobernadores que los frailes. El padre Valencia, por ejemplo, clérigo secular, sirvió como intérprete en dicha residencia de 1561 en la que se investigó al gobernador previo, don Luis de Céspedes de Oviedo, por parte del nuevo gobernador y juez de la residencia, doctor don Diego de Quijada. ${ }^{24}$

Para limitar el poder de los frailes, la corona creó otra institución como intermediario, el Juzgado Privado de los Indios de Yucatán, donde los intérpretes generales ocuparon un puesto muy destacado. La fundación de estas cortes, independiente de las de Nueva España, figura prominentemente entre las causas de esta disminución del poder de los franciscanos. Aunque durante los primeros años el obispo estuviera señalado como defensor de indios (desde 1579 hasta 1582), el desarrollo del juzgado de los indígenas significó la pérdida de gran parte del control directo que los frailes ejercían sobre los indígenas. ${ }^{25} \mathrm{~A}$ pesar de que algunos intérpretes y otros burócratas de este juzgado fuesen corruptos

\footnotetext{
${ }^{21}$ Lizana, Historia de Yucatán, edición Félix Jiménez Villalba. Madrid: Historia 16, 1988, pp. 119-126.

${ }^{22}$ Chuchiak, "The Indian Inquisition and the Extirpation of Idolatry: the Process of Punishment in the Provisorato de indios of the Diocese of Yucatan, 1563-1812" (Ph.D. Diss, Tulane University, 2000); González Cicero, Perspectiva religiosa en Yucatán, 1517-1571: Yucatán, los franciscanos y el primer obispo fray Francisco de Toral. México: El Colegio de México, 1978.

${ }^{23}$ Archivo General de las Indias (AGl), México 359, marzo 1567. Citada en Chuchiak, "In Servitio Dei: Fray Diego de Landa, the Franciscan Order, and the Return of the Extirpation of Idolatry in the Colonial Diocese of Yucatán, 1573-1579", The Americas 61, núm. 4 (April 2005): 611-46.

${ }^{24}$ Archivo General de la Nación (AGN), Inquisición 32, Libro 2, ff. 315-329.

${ }^{25}$ Borah, Justice by Insurance: The General Indian Court of Colonial Mexico and the Legal Aides of the Half-Real. Berkeley: University of California Press, 1983, pp. 354-355.
} 
e ineficientes, los funcionarios de esta corte ofrecieron un nuevo recurso para los indígenas ya que, efectivamente, muchos de los procesos que involucraron a los indígenas y que estuvieron apoyados por este tribunal, fueron pleitos contra curas.

\section{Intérpretes institucionalizados}

Fundado como respuesta práctica a la distancia geográfica y a la importancia de la población indígena, se desarrolló el Juzgado Privado de Indios de Yucatán, en un proceso de toma y daca entre los miembros de los consejos de los Austrias y los oficiales provinciales de Yucatán. Por un lado, este juzgado, autónomo de su homólogo en México, constituye un triunfo de las tendencias separatistas de Yucatán, mientras que por otra parte, representa una decisión lógica por parte del rey y sus consejeros al reconocer así la distancia con México (la capital virreinal), la diferencia de idiomas que separaba Yucatán de los territorios centrales conquistados anteriormente por los mexicas, y el poder sin paralelo de los frailes franciscanos. Debido a la supervivencia del idioma maya yucateco hasta finales de la época colonial y más allá, este tribunal pudo mantener su importancia y relevancia muchos más años que su homólogo en México central. ${ }^{26}$

Yucatán, una provincia con una integración al virreinato y, más tarde, a la nación mexicana, incompleta y disputada hasta el siglo xx, vio la formación de esta corte durante la década de los setenta en el siglo xvl, menos de treinta años después de que la corona decidiera definitivamente que Yucatán formara parte de Nueva España, en lugar de ser independiente o integrada en el Reino de Guatemala. Antes del año 1580 , se establecieron dos puestos esenciales, el intérprete general y el protector de los indios. A diferencia de los primeros protectores y defensores de los indios, el puesto de intérprete general no sufrió tanto movimiento de personal en sus primeros años. Desde los años ochenta hasta 1610, el noble maya Gaspar Antonio Chi sirvió como intérprete general, ganando un sueldo anual de ochenta pesos. ${ }^{27}$ Anteriormente, Gaspar Antonio Chi había trabajado como intérprete no oficial durante varios años, y lógicamente fue nombrado para el puesto cuando el rey decidió integrar los servicios de tres intérpretes como oficiales del nuevo Juzgado Privado de Indios de Yucatán. Chi nació antes de la conclusión de la conquista, y de alguna manera experimentó en muchos aspectos la vida típica de la elite maya en el siglo xv. Educado por los frailes,

${ }^{26}$ Muchas de las críticas hechas contra el Juzgado de México apuntaron que los indígenas de la metrópoli ya eran indistinguibles de las personas que pertenecian a las castas y españoles en términos de residencia e idioma. En Yucatán, la segregación idealizada no existía, pero la adopción de español se detuvo, o se invirtió. Por eso, la importancia de intérpretes en Yucatán no disminuyó con el transcurso del tiempo. Borah, Justice by Insurance, p. 391.

${ }^{27}$ Chuchiak, "To serve and protect: el juzgado general de indios y la defensoría indígena de Yucatán, 1591-1780", (s.f. manuscrito inédito). 
don Gaspar se convirtió en un personaje que gozó de autoridad y prestigio por toda la península, y no solamente a nivel local, como experimentaron la mayoría de los mayas pertenecientes a las elites durante la colonia a partir de principios del siglo xvir. Chi no solamente poseía la capacidad de escribir castellano y maya sino también latín y náhuatl, dándole una perspectiva más cosmopolita que la de muchos de sus sucesores. ${ }^{28}$

Gaspar Antonio Chi es conocido comúnmente como el único intérprete general maya. En un próximo artículo por publicar, Caroline Cunill observó que otros intérpretes mayas como Jorge Xiu, en 1562, y Agustín Ytza, "nahuatlato" (hablante de náhuatl), en 1573, servían con carácter provisional pocos años después de la conquista de Yucatán. ${ }^{29}$ Farriss también observó que don Fernando Uz, batab de Tekax, fue "intérprete oficial" en 1609, poco antes de que fuera implicado injustamente en la sublevación de Tekax de 1610, y que probablemente era el último de los intérpretes indígenas. ${ }^{30}$ Es posible que hubiera otros intérpretes mayas, como don Gaspar y don Fernando, sirviendo oficialmente o provisionalmente como traductores, pero lo que queda bien claro es que fueron españoles, algunos de ellos posiblemente mestizos, los que dominaron esa posición a partir de principios del siglo xvı. Al mismo tiempo, la presencia de hispanos en este puesto indica un aumento en el número de individuos laicos españoles capaces de hablar y escribir en maya. Aunque los líderes mayas perdieran su poder regional durante estos años, su poder local se mantuvo ya sea hasta finales del periodo colonial, o hasta los años precedentes a la Guerra de Castas, dependiendo de que análisis se encuentre más convincente, si el de Nancy M. Farris o el de Terry Rugeley, ambos prominentes historiadores. ${ }^{31}$

A pesar de su carácter extraordinario en términos de haber sido coautor de las primeras historias de Yucatán (Las relaciones geográficas), así como una figura multilingüe importante en toda la península, Gaspar Antonio Chi no se diferenciaba mucho de los intérpretes de la época colonial en sus diligencias cotidianas. En 1589, en un documento que forma parte del proceso de la inquisición contra Andrés Mexía, cura beneficiado, Chi tradujo quejas en su contra hechas por los mayas de su parroquia, siendo éste un trabajo continuo para los intérpretes de Yucatán, la traducción de quejas y reclamaciones contra curas. ${ }^{32}$ La existencia de la firma de don Pedro de Magaña como intérprete, pocas páginas después, indica que la estructura del juzgado privado, con dos intérpretes establecidos en

${ }^{28}$ Quezada y Okoshi Harada (eds.), "Introducción", Los papeles de los Xiu. México: UNAM, Instituto de Investigaciones Filológicas, 2001, p. 27, fn. 58.

${ }^{29}$ Cunill, "Alfabetización de los mayas yucatecos, 1545-1580", Temas Americanistas 20 (en prensa).

${ }^{30}$ Farriss, La sociedad maya, pp. 163-164. Farriss cita el AGI, Escribanía de cámara 305-A, Autos de Tekax, documentos que todavía no he podido ver para averiguar si Uz servía como Intérprete General formalmente nombrado por Decreto Real.

${ }^{31}$ Farriss escribió que el final del siglo xvı marcó el momento definitivo del declive de la elite maya, mientras que Rugeley propuso que su poder sobrevivió hasta el comienzo de la Guerra de Castas.

${ }^{32}$ AGN, Inquisición 69, Exp. 5, f. 275v. 
Mérida, ya había tomado forma. Debido a que era un oficio bilingüe, el puesto de intérprete recibía dos nombres, Intérprete General, en castellano, y Ah Tzol Than, "el cambiador de las palabras", en maya. El primer ejemplo de este término aparece con la rúbrica de Luis Cárdenas, en un documento de 1589, quien firmó bajo el nombre con su cargo, Ah Tzol Than.$^{33}$ Más de doscientos años después, Francisco Ruz también firmaría con este mismo título, en $1812 .^{34}$

\section{Los intérpretes del siglo xvII}

Al mismo tiempo que la importancia provincial de la elite de los mayas, como don Gaspar Antonio Chi y don Fernando Uz, disminuía, aumentaban el sueldo y las posibilidades de aprovecharse ilícitamente del puesto de intérprete general por parte de los españoles, quienes empezaron a monopolizar dicha posición. Don Francisco de Vargas se incluye como uno de los primeros españoles en ocupar dicho puesto, habiendo empezado a traducir oficialmente en 1600 al seguir los pasos de su antecesor don Pedro de Magaña. Chi había ganado ochenta pesos durante cada uno de los primeros diez años de su carrera, y terminó su vida obteniendo un sueldo de cien pesos, lo mismo que percibía Francisco de Vargas.

Por su parte, Manuel de Oliveras comenzó con el mismo sueldo que sus antecesores, cien pesos anuales, pero terminó su carrera de intérprete percibiendo doscientos pesos por año. ${ }^{35}$ Es difícil explicar cómo este aumento pudo coincidir con un descenso grave de la población indígena, ya que el sueldo era producto de un impuesto pagado por los mayas exclusivamente, conocido como el medio real o, en Yucatán, el Holpatán. Su nombre significa h'ol "adicional" y patan "impuesto".

El desarrollo de este puesto como una posición lucrativa probablemente también contribuyera a la exclusión de los mayas de ese cargo. Al haber más potencial de ganancia, había más competencia por el puesto. Fuera del sueldo oficial, existían otras maneras de aprovecharse financieramente, tanto legales como ilegales. Un manuscrito de John Chuchiak, todavía no publicado, se centra en un fraude cometido por don Pedro García de Ricalde, dándonos así un ejemplo de ganancia ilícita. ${ }^{36}$ Aunque en las situaciones extraordinarias también se producían oportunidades para ganancia lícita, por ejemplo cuando individuos bilingües servían como intérpretes durante visitas de obispos a sus arquidiócesis, o durante las residencias realizadas para enjuiciar la calidad del periodo de mandato de gobernadores salientes, o en incursiones en territorios habitados por mayas no

\footnotetext{
${ }^{33}$ Quezada y Okoshi Harada (eds.), Los papeles de los Xiu, p. 104.

${ }^{34}$ AGN, Tierras, v. 1419, Exp. 2, ff. 38-51, 92.

${ }^{35}$ Chuchiak, "To serve and protect: el juzgado general de indios y la defensoría indígena de Yucatán, 1591-1780," (s.f. manuscrito inédito).

${ }^{36} \mathrm{Id}$.
} 
conquistados. En estas ocasiones, los intérpretes podían ganar un sueldo mayor que su pago habitual.

Con una alta remuneración y la posibilidad de otras formas de obtener beneficios, el siglo xvı marcó una época en la que el cargo de traductor tenía un carácter prestigioso y provechoso. Manuel de Olivares, intérprete desde 1617 hasta 1640 , tipifica lo que sería un intérprete general del siglo xvı. Era encomendero de Tixcacal, Yalsihon y Ecab, con una encomienda cuyo valor era de cien pesos. ${ }^{37}$ Hasta finales de este siglo, por lo menos, algunos intérpretes combinaron sus trabajos de intérpretes con otras posiciones oficiales, como Francisco de Ávila (intérprete desde 1683-1690), quien recibía un sueldo de 200 pesos más las ganancias de su trabajo como escribano mayor de gobernación y guerra. ${ }^{38} \mathrm{Al}$ igual que Manuel de Olivares, su antecesor en ese siglo, Francisco de Ávila también fue encomendero. ${ }^{39}$

Los documentos fechados en el siglo xvII son más limitados e incompletos que los que nos han llegado del siglo xvin y de la primera mitad del siglo xix, pero aún sobreviven en número suficiente para darnos una idea básica del trabajo cotidiano de estos funcionarios. Al igual que sus homólogos del siglo xviI, estos también traducían peticiones, como la de don Juan Xiu Cimé de 1662. En este caso, Nicolás Cansino tradujo su petición de permiso para extender su privilegio de portar escopeta, con el motivo de ayudar a la aprehensión de indígenas huidos al "monte", o territorio fuera del control de los españoles. ${ }^{40}$ En 1688, el intérprete Francisco de Ávila confirmaría los mismos privilegios a favor de los Xiu, la familia a la cual pertenecía el anteriormente mencionado don Juan Xiu Cimé. ${ }^{41}$ En particular, vemos que intérpretes como Nicolás Cansino en 1662 y Francisco de Ávila en 1688 tradujeron peticiones para confirmar privilegios de la familia de la elite, los Xiu, una diligencia que sería continuada por sus sucesores en el siglo xvill. Los dos intérpretes anteriormente mencionados, Francisco de Ávila y Luis Cárdenas, se identificaron a sí mismos como ah tzol than cuando firmaron y tradujeron ese documento.

El papel de los intérpretes del siglo xvil se diferenció del de sus sucesores en que ellos también debieron actuar como embajadores ante varios grupos no conquistados o que habían huido del dominio español. Por ejemplo, Cristóbal Interián, quien aparentemente no era uno de los tres intérpretes generales oficiales, o por lo menos no aparece como tal en otros documentos, trabajó como "lengua" (otra palabra para intérprete en el uso colonial) durante la entrada de 1604 , en la "montaña". Cerca de la zona de los mayas yucatecos, Pablo Pax Bolon realizó el mismo papel de traductor frente a los habitantes de la región chontal

\footnotetext{
${ }^{37}$ García Bernal, Yucatán: Población y encomienda bajo los Austrias. Sevilla: Escuela de Estudios Hispano-Americanos, 1978, p. 545.

${ }^{38}$ Garcia Bernal, Yucatán bajo los Austrias, p. 180.

${ }^{39}$ Ibid., pp. 281-282.

${ }^{40}$ Papeles de los Xiu, f. 87.

41 lbid., p. 103.
} 
de Tabasco. ${ }^{42}$ Más tarde, Juan Carlos Maldonado y Juan Pascual de Aguirre de Campeche tradujeron el interrogatorio a Juan Aké de Bolonchén en 1668, quien representaba a Juan Yam, el cacique de unos mayas huidos "a la montaña", que es otra forma de referirse a la zona fuera del control colonial. ${ }^{43}$ Simultáneamente, la Iglesia llevó a cabo su propia campaña para reducir a los mayas sin el uso de la fuerza, con Br. don Cristóbal Ruiz sirviendo como "presbítero intérprete", quien se ocupó de traducir la declaración del huido regidor de Sahcabchén, Hernando Pech. ${ }^{44}$ Don Pedro García Ricalde, además de traductor $-\mathrm{y}$ a la vez defraudador de los indígenas como ya hemos comentado- escribió un minucioso informe sobre la situación de "la montaña" y los pueblos en sus cercanías en 1669. ${ }^{45}$ García Ricalde y el otro intérprete general, el sargento Nicolás Cardeña, también tuvieron mucho trabajo traduciendo las numerosas peticiones de los mayas huidos a la montaña. La necesidad de que los intérpretes debieran actuar como embajadores ante grupos de indígenas hostiles terminó mayormente después de la conquista de los itzaes.

\section{Los intérpretes del siglo xvIII y principios del xIX}

Aparte de este cambio, parece que las carreras de los intérpretes no cambiaron mucho entre el último siglo del reinado de los Austrias y el primer siglo con los Borbones en el poder, pero da la impresión de que el prestigio del puesto de intérprete podría haber experimentado un declive en el siglo xvil, ya que el sueldo no fue aumentado en todo ese periodo, (a partir de 1633 un sueldo de 200 pesos por año), y a finales de siglo una amplia variedad de individuos se encontraba sirviendo como intérpretes, sugiriendo mayor competencia para ocupar dicha posición entre numerosas personas bilingüies a finales de la época colonial. Un insulto dirigido a un intérprete en ese época, don Esteban de Castro, calificado como una persona de "linaje oscuro", indica también que los intérpretes del siglo xvil ya habían perdido el alto respeto que poseían sus antecesores. Además, aunque la encomienda seguía siendo la unidad básica de producción de la economía yucateca, hasta 1786, ningún encomendero sirvió como intérprete después de Francisco de Ávila, que había cumplido su periodo de servicio en 1690.

Los intérpretes del siglo xvin tuvieron cuatro tareas principales: traducir documentos de tierras, traducir peticiones (muchas veces quejas contra curas escritas por mayas), escribir bandos y otras órdenes oficiales en maya, e interpretar verbalmente en el transcurso de procesos legales entre mayas y españoles. Por la supervivencia de muchos más documentos del siglo xvin que de los siglos anteriores, especialmente en maya, podemos hacernos una mejor idea de los retos

\footnotetext{
${ }^{42}$ AGI, México 138.

${ }^{43}$ AGI, México 307 , ff. $7 \mathrm{v}$.

44 AGI, México 307.

${ }_{45}^{4} \mathrm{AGI}$, México 307 , ff. 2-3v.
} 
y la vida cotidiana de los intérpretes de aquella época, con casos de intérpretes en cada una de estas diligencias.

Por ejemplo, el papel esencial de los intérpretes en las transferencias de tierras se puede ver claramente en las ventas registradas en un legajo que incluye casi un siglo de documentos, que involucraron a cuatro intérpretes. En un proceso sobre el deslinde y la división y venta de propiedades de la Hacienda San Bernardo Chich, sirvieron sucesivamente los intérpretes generales don Juan Alonso de Rubio en 1735, Eusebio de Ayora en 1763, don Esteban de Castro durante la década de 1790 , y finalmente Francisco Ruz, alrededor de los últimos años de la Colonia, en $1815 .^{46}$ Debido a que el siglo xvil marcó un periodo durante el cual muchas tierras mayas cambiaron de manos, pasando a propietarios españoles, la asistencia de individuos bilingüies a estas ventas cobró más importancia hacia finales de la Colonia.

Fuentes documentales del periodo colonial revelan, desde los primeros escritos en maya en el alfabeto romano hasta los escritos de la época de la Independencia, los abusos de las autoridades españolas, que generaron numerosas quejas por parte de los indígenas contra los encomenderos, frailes, curas, y más tarde, contra los subdelegados y jueces españoles. Gracias a un ejemplo de estas quejas encontrado en unos documentos de finales de la época colonial, provenientes de Tetiz, podemos ver con buen detalle la actuación de los intérpretes en las demandas hechas por indígenas, en este caso en contra del cura don José María Domínguez y del cacique de dicho pueblo, don Simon Tuyu, por "malos tratos, imposiciones injustas, tareas, y el repartimiento". ${ }^{47}$ Luis Ruz, intérprete general, tradujo la queja original, mientras que don José María Pérez se encargó de las declaraciones verbales, lo cual sugiere que existía una división de labores a finales de la época colonial. Además, un intérprete del siglo xix, posiblemente pariente de Luis Ruz, Francisco Ruz, firmó al menos en una ocasión como ah tzol than hunluk (hun significa "papel" en maya), lo cual implica que estaba especializado en traducciones de textos y no en la traducción de declaraciones verbales. ${ }^{48}$ El abogado de los naturales, licenciado Diego de la Santa Cruz, citando una decisión de 1656, declaró que estas actuaciones del cura habían sido ilegales, y mandó a otro intérprete, don José María Pérez, a tomar testimonio del Teniente Eugenio Na, del Regidor Estevan Dzul y del "Alcalde de milpas" Laureano Yam. ${ }^{49}$ El intérprete Pérez está identificado en este proceso como intérprete de turno, dando así un título a los varios intérpretes que tradujeron de manera provisional. ${ }^{50}$ En otro caso, en 1790 , los principales del pueblo de Baca, Juan Noh,

${ }^{46}$ AGN, Tierras, v. 1419, Exp. 2, ff. 38-51, 92. En Papeles de los Xilu, Francisco Ruz aparece como intérprete general en 1812. "Trasunto del mandamiento hecho por Francisco Ruz, intérprete general", p. 167.

${ }^{47}$ AGN, Bienes Nacionales, v. 26; exp. 55, f. 2, tr. f. 4.

${ }^{48}$ Los papeles de los Xiu, p. 168.

${ }^{49}$ AGN, Bienes Nacionales, v. 26, Exp. 55, f. 3v-10v.

${ }^{50}$ AGN, Bienes Nacionales, v. 26, Exp. 55, f. 6v. 
batab, Clemente Cox y Bentura Ytza, alcaldes, Nicolás Koil, Juan de la Cruz Chim, Nicolás Ytza, Nicolás Poot, regidores, y Simon Canché, escribano, dirigieron una queja contra el trabajo forzado en la construcción de la iglesia — sin nombrar al cura que probablemente lo había impuesto-, la cual fue traducida al español por Vicente de Ávila. ${ }^{51}$

El asesinato del primer intendente de Yucatán, Lucas de Gálvez, generó ejemplos de los dos últimos tipos de trabajos que hemos nombrado, ejecutados por los intérpretes. En primer lugar, la veracidad de la coartada del reo principal, don Toribio del Mazo, sobrino del obispo y teniente de las milicias, se fiaba de las declaraciones de numerosos mayas y maya hablantes, los cuales en su mayoría lo contradijeron. Más de trescientos individuos fueron interrogados por más de nueve intérpretes. Específicamente, los testigos maya hablantes de Chikindzonot no confirmaron la presencia de don Toribio en dicho pueblo los días justo antes y después del asesinato. A pesar de una certificación, supuestamente firmada por todos los principales confirmando su presencia en Chikindzonot, ningún testigo entrevistado por los intérpretes Eugenio Cano, Mariano Ancona y don José Joaquín Pérez, confirmó que se hubiera encontrado allí. Asimismo, bandos publicados originalmente en español pidiendo informes sobre viajes de incógnito u otro tipo de actividades sospechosas, tuvieron que ser traducidos al maya por el intérprete general don Esteban de Castro para poder comunicar la información en la lengua franca de la provincia.

A veces los intérpretes no se ganaban la confianza de sus superiores, que eran exclusivamente hispanohablantes, y la causa de esta desconfianza fue el hecho del potencial que poseían los intérpretes para abusar de su poder, por ejemplo malinterpretando o influyendo en los testigos. Eugenio Cano, un garzón de la plana mayor de blancos de la división de milicias pardas de Yucatán, sirvió en muchas ocasiones como intérprete cuando los dos intérpretes oficiales, Vicente de Ávila y Esteban de Castro no se encontraban disponibles. Parece que este soldado no solamente hablaba maya lo suficientemente bien como para servir de intérprete provisional, sino que aparentemente también hablaba francés. Una comunidad fundada por insurgentes francófonos durante la rebelión, de 1789, contra Francia en Santo Domingo — más tarde aliados de España - recibió el derecho de refugiarse en Yucatán. Estos formaban una población de 115 individuos, probablemente todos francófonos, en un asentamiento en su mayor parte libre de supervisión por parte del gobierno provincial. De modo interesante, en su libro sobre la comunidad afroamericana de San Fernando Aké, Jorge Victoria Ojeda y Jorge Canto Alcocer han observado que el sargento Cano fue el primer intérprete de la comunidad, habiendo trabajado como intérprete de los mayas y también con otro grupo de afroamericanos, los pardos, donde servía como oficial. Es impresionante que un hombre sin educación formal, sin

${ }^{51}$ Archivo General del Estado de Yucatán (AGEY), Ramo Colonial, Sección Ayuntamientos, vol. 1, exp. 3 , ff. $1-10$. 
el derecho a utilizar el título de "don", que marcaba la pertenencia a un estatus de elite, fuera capaz de escribir, leer y hablar en maya, español y probablemente también en francés.

Al no poseer la habilidad de hablar maya, burócratas y oficiales militares de otras provincias o países ponían en duda la veracidad de los intérpretes que pudieran tener vínculos políticos, o familiares involucrados en procesos criminales, u otros intereses en dichos procesos. El doctor don Francisco de Guillén, el juez comisionado y sin duda el más perseverante entre los funcionarios encargados de identificar y procesar a un reo por el asesinato de don Lucas, confió en las habilidades de Cano como traductor provisional, debido a la ausencia de los dos intérpretes generales oficiales. Anteriormente Cano ya había servido como guardia del mismo reo principal, don Toribio, y a causa de la conflictiva relación entre el carcelero y el reo, la veracidad de las traducciones hechas por Cano fueron puestas en duda. Cano también declaró varias veces en la documentación del proceso contra el asesino de Gálvez, abiertamente convencido de la culpabilidad del sobrino del obispo. A su vez, don Toribio notó ciertos defectos en los procedimientos de Cano, variaciones del protocolo normal de los intérpretes, como por ejemplo que, en contra de las leyes, Cano pasara por Sotuta traduciendo declaraciones de varios maya hablantes sin otro intérprete presente, como era costumbre. Debido a las sospechas de que esas traducciones no fueran fieles y de que influyera en los testigos, don Toribio le calificó como un "influidor de testigos". 52

Por otro lado, hubo otro intérprete que tuvo vínculos familiares con don Toribio, o por lo menos, con la casa del obispo. El doctor Guillén, que observaba la sociedad con más perspicacia que los demás jueces comisionados, notó que don Enrique González, además de ser intérprete en varias actas del proceso para la averiguación del asesino, era también compadre de don Francisco Ortiz. Ortiz a su vez sirvió como mayordomo del palacio episcopal y más tarde como defensor de don Toribio. En su declaración, don Pedro Rivas de Rocafull observó muchas conversaciones entre los dos compadres, dejando bajo sospecha las actuaciones de don Enrique. ${ }^{53}$ Para añadir más razón a estos recelos, don Enrique había actuado como uno de los dos "peritos" que averiguaron la autenticidad de la certificación de la coartada de don Toribio.

\section{El siglo xvill y xix: ¿un periodo de aumento del idioma maya?}

Un mínimo de trece intérpretes se encontraban de servicio en distintos lugares durante los cuatro primeros años del proceso contra don Toribio del Mazo, sos-

\footnotetext{
${ }^{52}$ AGN, Criminal 392, f. 411

${ }_{53}$ AGI, México 3040, f. 60.
} 
pechoso del asesinato del primer intendente. ${ }^{54}$ Además, algunos títulos como ah tzol than hunluk, e intérprete de turno, sugieren que hubo más trabajo y al mismo tiempo más individuos calificados para trabajar en estas diligencias de traducción. El impresionante número de intérpretes que participaron en este proceso como traductores de documentos, intérpretes de testigos maya hablantes, y peritos, cuya labor sería asegurar la autenticidad de los escritos mayas, demuestra que el número de personas de herencia no maya con la capacidad de leer y escribir en el idioma natural de la península, a finales del siglo xvin no había disminuido, sino que posiblemente se encontraba en aumento.

¿Cómo aprendieron los españoles el idioma maya? Aparte del contacto cotidiano con los mayas que tenían los curas y frailes, también hubo otro tipo de españoles que pasaron mucho tiempo comunicándose con la población como parte de su trabajo, por ejemplo soldados y encomenderos. Más tarde, con la abolición de la encomienda y la ordenanza de intendentes de 1786, mayordomos de haciendas, jueces españoles y subdelegados también estarían en contacto continuo con la población indígena. De los once intérpretes mencionados, por ejemplo, Mariano Ancona sirvió como Cabo de Granaderos y juez español, Cano como sargento o garzón de las milicias pardas, y Manuel de Aguilar sirvió como teniente de milicias. En la huida clandestina de don Toribio de Mérida después del asesinato, hecho presenciado por decenas de mayas, don Toribio utilizó los servicios de su mayordomo, don Nicolás Domínguez, quien había aprendido maya durante su residencia en la hacienda Balix.

\section{La sociedad vista por las obras de los intérpretes}

La situación lingüística de Yucatán a finales del periodo colonial había cambiado, como se puede comprobar gracias a las muchas obras realizadas por intérpretes. Pero también el hecho de residir en una zona urbana o rural podía ser muy determinante, ya que a pesar de su etnia, los mayas en la ciudad podían escribir y hablar castellano en mucho mayor número que sus homólogos rurales, mientras que muchas personas pertenecientes a otros grupos — castas - de fuera de Mérida, únicamente hablaban maya. En Mérida, por ejemplo, un "indio ladino", Isidoro Xix, sirvió como pregonero de Mérida, publicando informes sobre ventas públicas en la década de $1790 .^{55}$ Otro "indio ladino", Salvador Pech, escribió sus propias cartas de venta en español en $1792 .{ }^{56}$ En una venta de un solar en Mérida, una mujer maya, María Candelaria Ek, también escribió su carta de venta de 1782 en el idioma europeo. ${ }^{57}$ Finalmente, el cabildo y el escribano del Barrio

\footnotetext{
${ }^{54}$ AGN, Criminal volúmenes 302, 316, 317, 332 y 335; AGI, México volúmenes 3036 y 3040.

55 Archivo Notarial del Estado de Yucatán (ANEY), Libro 29, 1791-92, Cuaderno 1, ff. 18, 24; ANEY Libro 31, 1794, Cuaderno 1, ff. 11-12.

56 ANEY Libro 29, 1791-92, Cuaderno 10, ff. 284-285.

57 ANEY Libro 28, 1790, Cuaderno 2, f. 302.
} 
de Santiago de Mérida presentaron una carta de venta de 1799 en castellano, firmada por el escribano, José Venancio Chan. ${ }^{58}$

Para un buen contraste con la situación histórico-lingüística de Mérida, a finales del siglo xvin, podemos repasar la gran cantidad de información sobre Chikindzonot que ha llegado a nuestras manos por casualidad. Por su importancia durante la averiguación de la coartada de don Toribio, quien pidió tres certificaciones en dos idiomas confirmando su presencia allí durante el asesinato, este pueblo remoto y normalmente fuera de la vista de los españoles, cayó bajo la mirada de las autoridades por muchos años. Chikindzonot, hoy ubicado en la frontera con Quintana Roo, y uno de los pueblos que iniciaron la Guerra de Castas, aparece en alto relieve en un volumen completo y en gran parte de otro como resultado del proceso contra don Toribio. En estas páginas, además de ver que la coartada fue un complot del cura, don Toribio, y algunos otros aliados, podemos ver una situación muy distinta de la de Mérida, una situación donde el idioma maya prevalecía entre casi todos los residentes, y donde incluso la viuda e hija del juez español hacían declaraciones en maya. ${ }^{59}$

Para los españoles en zonas rurales, el aprendizaje del idioma maya era necesario para la vida cotidiana, como demuestran papeles confiscados a varios funcionarios y clérigos locales. Como hemos visto, el criado de don Toribio, don Nicolás Domínguez, aprendió maya mientras trabajaba como mayordomo en la hacienda de su amo. Los dos curas de Chikindzonot, don Manuel Correa y, después de su prisión, don Jacinto Rubio, escribieron varios documentos en maya. ${ }^{60}$ En los papeles confiscados del fallecido juez español se incluyen muchos documentos en maya, cuatro de ellos descritos en detalle en el proceso, que fueron escritos por don Gregorio Yama, el cacique, Paulino Canché y Eulogio Tomas. ${ }^{61}$ Este último nos sugiere que aún entre individuos de la teórica república de españoles residentes en el campo, el maya era el idioma predilecto.

Por una breve descripción escrita por el juez subdelegado de Tihosuco, don Mateo de Cárdenas, sabemos que Chikindzonot tuvo una población impresionantemente diversa para un pueblo aislado. Según este escritor, Chikindzonot "se ha compuesto siempre de Indios, y unos sinquenta Vessinos de otras Castas, en la mayor parte mulatos [...] cuya lengua hablan únicamente."62 También eran pocos los españoles que vivían allí, entre ellos el cura don Manuel Correa, y después el juez español. Pero, por estar bien claro quiénes eran indígenas y quiénes los pocos españoles, sabemos que los residentes con apellidos hispanos probablemente pertenecían a las mencionadas "castas", mayormente de herencia africana. Felipe Santos, Pablo Santos, Nicolás Santos, Lorenzo Gomes - los

\footnotetext{
${ }^{58}$ Archivo Histórico del Arquidiócesis de Yucatán (AHAY), Asuntos terminados, vol. 6 (1788-1792), $\mathrm{s} / \mathrm{n}$.

${ }^{59}$ AGN, Criminal 332, 58 .

${ }^{60}$ AGN, Criminal 335 , varios.

${ }^{61}$ AGN, Criminal 316, Cuaderno 48, f. 6

${ }^{62}$ AGN, Criminal 335, f. 39.
} 
primeros testigos examinados- probablemente provenían de este grupo. Todos hablaban maya, encargándose de la traducción Mariano Ancona. ${ }^{63}$ Otros, incluso Sebastián Santos, Gregorio Santos, José Santos, Honorato Gonzáles, Juan Santos, Jesús Gomes, Carlos Gomes, Froilán Santos, no eran capaces de hablar español ni siquiera a nivel básico. El único de estas castas que mostró aptitud -aunque limitada - para hablar en castellano fue Laureano Rivero, asimismo el único de las "castas" que firmó su declaración. Don Mateo de Cárdenas, subdelegado del partido de Tihosuco, escribió que "se examinó con intérprete por estar torpe en la lengua castellana y diestro en la maya". ${ }^{64}$ Laureano Rivero también se diferenció por su trabajo como sastre de la mayoría de los residentes de Chikindzonot, los cuales, según el mencionado cura, "pasan una vida miserable con el escaso lucro de una milpa corta de maíz, que trabaja por su proprio mano". ${ }^{65}$ Rivero había viajado fuera de su pueblo, trabajando también en Yaxcabá. Probablemente había aprendido castellano debido a sus viajes, experiencias que le habían expuesto a más interacciones con españoles que a la mayoría de los residentes de Chikindzonot.

Estas castas, aparte de ser representativas de la cultura española en un medio indígena, habían absorbido la cultura material y el idioma de los habitantes originales de la península. Si esto no es muy sorprendente, sí lo es el hecho de que individuos provenientes del aparato de gobernación del campo también hablaran únicamente maya. Por ejemplo, el sargento de las milicias urbanas de Chikindzonot, Manuel Rejón, necesitó un intérprete por ser "muy poco versado en el castellano, y saber muy bien la lengua maya”. ${ }^{66}$ Aún más interesante, Tomasa Santos, la viuda de don Pedro Gutiérrez, juez español del pueblo, y su hija, cuando murió Gálvez hicieron sus declaraciones en maya. ${ }^{67}$ Esta situación, que se producía - al mismo tiempo que los españoles, supuestamente, cumplían su "segunda conquista"- en la familia misma de este agente de hispanización, demuestra las limitaciones de tal segunda conquista.

\section{Conclusiones}

La persistencia del idioma maya entre la comunidad indígena de Guatemala representó una forma de resistencia, como ha observado Robert M. Hill en su libro sobre los cakchiqueles. ${ }^{68}$ Del mismo modo, esta forma de resistencia también

\footnotetext{
${ }^{63}$ AGN, Criminal 332, ff. 3-6.

${ }^{64}$ AGN, Criminal 332 , f. $10 \mathrm{v}$.

${ }^{65}$ AGN, Criminal 335, f. 39.

${ }^{66}$ AGN, Criminal 335, f. 29.

${ }^{67}$ AGN, Criminal 316 , f. 67.

${ }^{68}$ Hill, Colonial Cakchiquels: Highland Maya Adaptation to Spanish Rule 1600-1700. Fort Worth, Texas: Harcourt Brace Jovanovich, 1992. El capítulo 8 trata de la persistencia del idioma cakchiquel escrito como rechazo al español.
} 
existía en Yucatán. Por ejemplo, el criado, cochero, y a veces zapatero, Luis Lara contestó en castellano durante sus conversaciones extrajudiciales, pero se negó a hablar en castellano tan pronto como empezaron los procedimientos oficiales. Conocedor de sus derechos ante la ley, Lara sabía que tenía derecho a la presencia de un intérprete para declarar en su idioma preferido. Asimismo, podemos decir que la presencia de maya hablantes en gran parte de la población no indígena, representa un ejemplo del frecuentemente negado poder que tuvieron los mayas de Yucatán para influir en la supuesta "cultura dominante".

Pero como hemos visto, esta influencia fue más poderosa en el campo que en la ciudad. Terry Rugeley y Wolfgang Gabbert han observado que en los años precedentes a la Guerra de Castas la división entre el campo y la ciudad no era idéntica a la división entre los mayas y los españoles propuesta por Farriss. Este hecho se puede ver claramente en los procesos jurídicos que involucraban intérpretes. El tribunal de los indios no cambió en su estructura, pero sus clientes no eran los mismos que cuando el juzgado se fundó. Individuos como Salvador Pech y María Candelaria Ek, los vendedores de tierras previamente mencionados, hicieron sus transacciones sin apoyo ni asistencia de los cabildos indígenas ni de los burócratas del Juzgado Privado de Indios de Yucatán; mientras que casi la totalidad de residentes en Chikindzonot, incluyendo a todas las castas, e incluso a la viuda e hija del juez español, el funcionario europeo de más alto rango, necesitaron de la asistencia de un intérprete. ${ }^{69} \mathrm{~A}$ pesar de eso, los intérpretes siguieron ganando su sueldo a partir del impuesto anteriormente mencionado, el holpatán. A finales de la época colonial algunos pagaban este impuesto sin tener necesidad de intérpretes, mientras que otros que se aprovechaban de sus servicios no tenían que pagarlo.

Es necesario descartar las dos ideas generalmente aceptadas para explicar la situación histórico-linguiística de Yucatán a fines de la Colonia. En primer lugar, no existía una división rígida entre la mayoría indígena y la minoría europea. Numerosos individuos tanto de herencia indígena como europea cruzaron la teórica barrera cultural y linguiística que supuestamente dividió las dos repúblicas, incluyendo a los intérpretes y también a los indios ladinos de Mérida. Estos individuos son representativos del "medio bilinguie" que era Yucatán, descrito por Alfredo Barrera Vásquez. Además, un creciente grupo de personas que no eran ni españoles ni indígenas vino a complicar la situación, como las castas de Chikindzonot, que todas ellas hablaban únicamente maya. Como lo demuestran tanto la existencia de castas monolingüies en maya, como los criados indígenas residentes en hogares españoles, que hablaban castellano, la división entre las dos repúblicas propuestas no era fija.

Hasta ahora los historiadores han prestado gran atención a los cambios resultado de la influencia española. Sin embargo, puede ser que siguiendo los pasos de Barrera Vásquez seamos capaces de comprender mejor el pasado de Yucatán y lo

${ }^{69}$ AGN, Criminal 332 , varios. 
consideremos como un proceso de intercambio entre los mayas, españoles y africanos, más que desde la perspectiva teleológica que describe una completa occidentalización. Dejando atrás esta tradicional perspectiva, una nueva manera de mirar el pasado podría revelar una situación similar a la sociedad colonial descrita en la revisionista historia escrita por Colin MacLachlan y Jaime E. Rodríguez O. Según estos autores, durante la historia colonial de Nueva España no se dio un movimiento hacía una mayor europeización, sino que durante aquella época se desarrolló una cultura "mestiza", que no era ni indígena ni española. ${ }^{70}$ Con este nuevo paradigma, podremos entender a la sociedad yucateca en su conjunto, y especialmente las interacciones entre los grupos previamente estudiados de manera individual.

\section{BIBLIOGRAFIAA}

Antochiw, Michel

2006 Milicia de Yucatán (siglos xv y xvul) \& La unión de armas de 1712. México: INAH, CONACULTA.

\section{Barrera Vásquez, Alfredo}

1980-1981 Estudios lingüísticos. Mérida: Fondo Editorial de Yucatán.

[1948] La lengua maya y su influencia en el español de Yucatán, manuscrito inédito, Tulane University, Latin American Library Rare Books.

Borah, Woodrow

1983 Justice by Insurance: The General Indian Court of Colonial Mexico and Legal Aides of the Half-Real. Berkeley: University of California Press.

Campos García, Melchor

2005 Castas, feligresía y ciudadanía en Yucatán: los afromestizos bajo el régimen constitucional español, 1750-1822. Mérida: CONACYT/UADY.

Chuchiak, John F.

2000 "The Indian Inquisition and the Extirpation of Idolatry: the Process of Punishment in the Provisorato de indios of the Diocese of Yucatan, 1563-1812". Tesis de doctorado, Tulane University, Nueva Orleans.

2005 "In Servitio Dei: Fray Diego de Landa, the Franciscan Order, and the Return of the Extirpation of Idolatry in the Colonial Diocese of Yucatán, 15731579," The Americas 61, núm. 4.

s. f. "To Serve and Protect: El juzgado general de indios y la defensoría indígena de Yucatán, 1591-1780", manuscrito inédito.

\footnotetext{
${ }^{70}$ MacLachlan y Rodríguez O., The Forging of the Cosmic Race: A Reinterpretation of Colonial Mexico. Berkeley: University of California Press, 1990, p. 3.
} 
Coe, Michael y Justin Kerr

1998. The Art of the Maya Scribe. Nueva York: Harry N. Abrams, 1998.

Colazo Simón, Antonia

2007 "Los fenómenos glotales en el español hablado en Yucatán", ponencia presentada en el VII Congreso de Mayistas, julio 10, 2007. Mérida, Yucatán.

Cunill, Caroline

(en prensa) "Alfabetización de los mayas yucatecos, 1545-1580", Temas Americanistas 20.

Delvendahl, Kai

2006 "Las sedes del poder: arquitectura, espacio, función y sociedad de los conjuntos palaciegos del Clásico Tardío en el área maya, evaluados desde la arqueología y la iconografía". Tesis de doctorado, UNAM, Facultad de Filosofía y Letras, México.

Díaz del Castillo, Bernal

2005 Historia verdadera de la conquista de la Nueva España, edición crítica José Antonio Barbón Rodríguez. México: El Colegio de México.

Dumond, Don E.

1997 The Cross and the Machete: Campesino Rebellion in Yucatan. Lincoln: University of Nebraska Press.

Espejo Ponce-Hunt, Marta

1974 "Colonial Yucatán: Town and Region in the Seventeenth Century". Tesis de doctorado, UCLA, Los Ángeles.

Farriss, Nancy M.

1984 Maya Society under Colonial Rule: The Collective Enterprise of Survival. Princeton, N. J.: Princeton University Press.

Gabbert, Wolfgang

2004 Becoming Maya: Ethnicity and Social Inequality in Yucatán since 1500. Tucson: University of Arizona Press.

2004 "Of Friends and Foes: The Caste War and Ethnicity in Yucatan", The Journal of Latin American Anthropology 9, núm. 1: 90-118.

García Bernal, Manuela Cristina

1978 Yucatán: población y encomienda bajo los Austrias. Sevilla: Escuela de Estudios Hispano-Americanos.

González Cicero, Stella María

1978 Perspectiva religiosa en Yucatán, 1517-1571: Yucatán, los franciscanos y el primer obispo fray Francisco de Toral. México: El Colegio de México. 
Lipski, John

2005 A History of Afro-Hispanic Language: Five Centuries, Five Continents Cambridge: Cambridge University Press.

Lizana, Bernardo de

1988 Historia de Yucatán, edición Félix Jiménez Villalba. Madrid: Historia 16.

Ojeda, Jorge Victoria y Jorge Canto Alcocer

2006 San Fernando Aké: microhistoria de una comunidad afroamericana en Yucatán. Mérida: UADY.

Quezada, Sergio y Tsubasa Okoshi Harada (eds.)

2001 Los papeles de los Xiu. México: UNAM, Instituto de Investigaciones Filológicas, Centro de Estudios Mayas.

Restall, Matthew

2004 "Maya Ethnogenesis", The Journal of Latin American Anthropology 9, núm. 1: 64-89.

1997 The Maya World: Yucatec Culture and Society, 1550-1850. Stanford: Stanford University Press.

(en prensa) The Black Middle.

y John F. Chuchiak IV

2002 "A Reevaluation of the Authenticity of Fray Diego de Landa's Relación de las cosas de Yucatán", Ethnohistory 49, núm. 3: 651-669.

Rugeley, Terry

1996 Yucatan's Maya and the Origins of the Caste War. Austin: University of Texas Press.

\section{Documentación de archivo}

- Archivo General de la Nación (México, D. F), Secciones Criminal, Inquisición y Bienes Nacionales.

- Archivo General de las Indias (Sevilla, España), Audiencia de México.

- Archivo Notarial del Estado de Yucatán (Mérida, Yucatán), Libros de Protocolos 28. 29 y 31.

- Archivo General del Estado de Yucatán (Mérida, Yucatán), Ramo Colonial.

- Archivo Histórico de la Arquidiócesis de Yucatán (Mérida, Yucatán), Asuntos Terminados. 